\title{
Predicting Outcomes of In-Hospital Cardiac Arrest: Retrospective US Validation of the Good Outcome Following Attempted Resuscitation Score
}

\author{
Jeffrey B. Rubins, $M D^{7}$, Spencer D. Kinzie, $M D^{2}$, and David M. Rubins, $M D^{3}$ \\ 'Palliative Care Division, Hennepin Healthcare, University of Minnesota, Minneapolis, MN, USA; ${ }^{2}$ Division of General Internal Medicine, Hennepin \\ Healthcare , University of Minnesota, Minneapolis, MN, USA; ${ }^{3}$ Department of Medicine, Brigham and Women's Hospital, Harvard Medical School, \\ Boston, MA, USA.
}

BACKGROUND: Providers should estimate a patient's chance of surviving an in-hospital cardiac arrest with good neurologic outcome when initially admitting a patient, in order to participate in shared decision making with patients about their code status.

OBJECTIVE: To examine the utility of the "Good Outcome Following Attempted Resuscitation (GO-FAR)" score in predicting prognosis after in-hospital cardiac arrest in a US trauma center.

DESIGN: Retrospective observational study

SETTING: Level 1 trauma and academic hospital in Minneapolis, MN, USA

PARTICIPANTS: All cases of pulseless in-hospital cardiac arrest occurring in adults (18 years or older) admitted to the hospital between Jan 2009 and Sept 2018 are included. For patients with more than one arrest, only the first was included in this analysis.

MAIN MEASURES: For each patient with verified inhospital cardiac arrest, we calculated a GO-FAR score based on variables present in the electronic health record at time of admission. Pre-determined outcomes included survival to discharge and survival to discharge with good neurologic outcome.

KEY RESULTS: From 2009 to 2018, 403 adults suffered in-hospital cardiac arrest. A majority (65.5\%) were male with a mean age of 60.3 years. Overall survival to discharge was 33.0\%; survival to discharge with good neurologic outcome was $17.4 \%$. GO-FAR score calculated at the time of admission correlated with survival to discharge with good neurologic outcome (AUC 0.68), which occurred in $5.3 \%$ of patients with below average survival likelihood by GO-FAR score, $22.5 \%$ with average survival likelihood, and $34.1 \%$ with above average survival likelihood.

CONCLUSIONS: The GO-FAR score can estimate, at time of admission to the hospital, the probability that a patient will survive to discharge with good neurologic outcome after an in-hospital cardiac arrest. This prognostic information can help providers frame discussions with patients on admission regarding whether to attempt cardiopulmonary resuscitation in the event of cardiac arrest.

This paper has not been presented at any conference.

Received December 17, 2018

Revised May 15, 2019

Accepted August 7, 2019

Published online September 11, 2019
KEY WORDS: in-hospital cardiac arrest; GO-FAR; code status.

J Gen Intern Med 34(11):2530-5

DOI: $10.1007 / \mathrm{s} 11606-019-05314-\mathrm{x}$

(C) Society of General Internal Medicine 2019

\section{INTRODUCTION}

In-hospital cardiac arrest (IHCA) affects an estimated 209,000 patients in the USA annually, with an overall rate of survival to discharge most recently estimated at $25.8 \% .^{1}$ Hospitals are required to elicit patient choices at time of hospital admission regarding end-of-life events, including choices about treatment for IHCA, in accordance with the Patient SelfDetermination Act of $1990 .{ }^{2}$ Discussions with patients about the benefits and risks of cardiopulmonary resuscitation (CPR) are a form of informed consent, with unique aspects in that the discussion takes place typically well in advance of the potential need for the procedure, and that the default is to perform the procedure. In many teaching hospitals, the admitting provider, often a provider-in-training, is responsible for this discussion and for entering an order to perform ("Full Code") or not perform ("Do Not Resuscitate, DNR") CPR in the event of IHCA. Providers are responsible for presenting information to patients about the likelihood of outcomes after CPR, including quality of life and survival, in order to participate in shared decision making. However, providers often fail to give patients this information and are reluctant to provide a recommendation to patients about whether CPR should

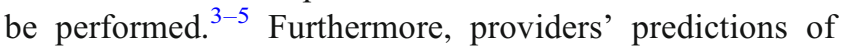
probability of survival after CPR may not be accurate. ${ }^{4}$

Therefore, providers need a tool that is accessible at time of admission to supply prognostic information regarding whether a patient is likely to benefit from CPR for IHCA and specifically, their chance of surviving to discharge with intact cognitive function. Several pre-arrest scoring systems have been developed in the past; ${ }^{6,7}$ however, these did not have sufficient predictive accuracy nor an assessment of a patient's neurological and cognitive function at discharge, and included variables that were not available at the time of admission. 
Table 1 GO-FAR Score Determined at Hospital Admission

\begin{tabular}{l}
\hline Variable \\
\hline $\begin{array}{l}\text { Neurologically intact at } \\
\text { admission (CPC }=1) \\
\text { Major trauma }\end{array}$ \\
Acute stroke \\
Metastatic or \\
hematologic cancer \\
Septicemia \\
Medical non-cardiac di- \\
agnosis \\
Hepatic insufficiency* \\
Admitted from skilled \\
nursing facility \\
Hypotension or \\
hypoperfusion $\dagger$
\end{tabular}

Renal insufficiency or dialysis*

Respiratory insufficiency

\footnotetext{
Age, years$$
<70
$$$$
\begin{aligned}
& <70 \\
& 70-74
\end{aligned}
$$$$
75-79
$$$$
80-84
$$$$
\geq 85
$$

Definition
See Table 2
Multisystem injury or single-
system injury associated with
shock or altered mental status
Diagnosis of intracranial or
intraventricular hemorrhage or
thrombosis
Solid tissue malignancy with
metastasis or any hematologic
malignancy
Active bloodstream infection
Total bilirubin $>2$ mg/dL and
AST $>2$ times the upper limit of
normal, or cirrhosis

$\mathrm{SBP}<90$ or $\mathrm{MAP}<60 \mathrm{mmHg}$; vasopressor or inotropic requirement after volume $\leq 3 \mu \mathrm{g} / \mathrm{kg} / \mathrm{min})$; or intra-aortic balloon pump

Serum creatinine $>2 \mathrm{mg} / \mathrm{dL}$ or ongoing dialysis/ extracorporeal filtration therapies

Any of the following: $\mathrm{PaO} 2 / \mathrm{FiO} 2$ ratio $<300, \mathrm{PaO} 2<60 \mathrm{mmHg}$, or $\mathrm{SaO} 2<90 \%$ (without preexisting cyanotic heart disease); $\mathrm{PaCO} 2>$ $50 \mathrm{mmHg}$; spontaneous respiratory rate $>40 / \mathrm{min}$ or $<5 / \mathrm{min}$; or requirement for artificial ventilation (negative pressure, or positive pressure either noninvasive, or via invasive airway) Any of the following: $\mathrm{PaO} 2 / \mathrm{FiO} 2$ ratio < $300, \mathrm{PaO} 2<60 \mathrm{mmHg}$, or $\mathrm{SaO} 2$ $<90 \%$ (without preexisting cyanotic heart disease); $\mathrm{PaCO} 2>$ $50 \mathrm{mmHg}$; spontaneous respiratory rate $>40 / \mathrm{min}$ or $<5 / \mathrm{min}$; or requirement for artificial ventilation (negative pressure, or positive pressure either noninvasive, or via invasive airway)

Antibiotic therapy has not yet been started or is still ongoing expansion (except for dopamine

Score

$-15$

10

8

7

7
Modified from Ebell et al. ${ }^{8}$

$A S T$, aspartate aminotransferase; FiO2, fraction of inspired oxygen: $M A P$, mean arterial pressure; SaO2, arterial oxygen saturation; $S B P$, systolic blood pressure

$*$ Determined with 24 h of cardiac arrest in original study ${ }^{8}$

tDetermined within $4 \mathrm{~h}$ of cardiac arrest in original study ${ }^{8}$

The "Good Outcome Following Attempted Resuscitation" (GO-FAR) score was developed using data available from the Get With the Guidelines American Heart Association Resuscitation registry on 51,240 patients suffering an IHCA between 2007 and 2009. ${ }^{8}$ Using 13 variables (Table 1), the GO-FAR score accurately predicted the likelihood of survival to discharge with good neurologic outcome following IHCA in the internal validation split sample. Since this publication, two studies in Sweden (287 adults with IHCA in 2007-2010 at Skåne University Hospital ${ }^{9}$ and 717 adults with IHCA in 2013-2014 in six hospitals in Stockholm County ${ }^{10}$ ) have externally validated the utility of the GO-FAR score. In this study, we not only report the first external validation in a US hospital of the GO-FAR score for predicting patient outcomes after IHCA events but also investigate its performance when calculating the score at the time of admission.

\section{METHODS}

\section{Setting and Study Population}

This is a retrospective observational study including all patients 18 years and older suffering pulseless in-hospital cardiac arrest (IHCA) receiving cardiopulmonary resuscitation (CPR) between January 2009 and September 2018 at Hennepin County Medical Center (Hennepin Healthcare, Minneapolis, $\mathrm{MN}$ ), a nationally recognized 440-bed level 1 trauma center, academic training hospital, and safety-net hospital. The Institutional Review Board of the Minnesota Medical Research Foundation/Hennepin Healthcare approved this study and waived the requirement for informed consent.

\section{Data Sources}

Cases were identified by a search of the electronic health record (Epic Systems Corporation, Madison, WI) for "Code Blue" notes (which are by hospital protocol entered into the health record upon activation of the Medical Emergency Response Team) and compared with a database maintained by the hospital quality performance improvement team. Of the 1326 Code Blue alert notes retrieved, 923 were excluded due to the following reasons: 697 were not pulseless cardiac arrests, 181 were out-ofhospital cardiac arrests presenting to the emergency department, 5 were patients $<18$ years of age, 30 were initiated in patients with DNR orders and stopped, 9 had subsequent cardiac arrests wherein only the first instance was used in 3 patients, and 1 was a patient on venousvenous extracorporeal membrane oxygen support at time of the arrest. A total number of 403 cases remained for further analysis.

Two of the authors independently calculated the GO-FAR score for each case, according to the presence or absence of the variables at time of hospital admission, using an online calculator (available at MDCalc [www.mdcalc.com]). In cases where the calculated scores differed, the lead author reviewed the record and reconciled any differences. The variables included in the score calculation were the same as those derived and validated by Ebell and colleagues, ${ }^{8}$ with one exception. Given our goal to use the GO-FAR score at the time of admission to the hospital to predict the outcome of CPR for IHCA, several of the variables which Ebell et al. ${ }^{8}$ defined as occurring at 4-24 $\mathrm{h}$ prior to the event were determined instead 
Table 2 Cerebral Performance Category (CPC) Score

\begin{tabular}{ll}
\hline \hline CPC & Description \\
\hline 1 & $\begin{array}{l}\text { Alert, able to work, and lead a normal life. May have minor } \\
\text { psychologic or neurologic deficits }\end{array}$ \\
2 & $\begin{array}{l}\text { Conscious, able to work in sheltered environment, able to } \\
\text { perform independently activities of daily life. May have } \\
\text { hemiplegia, seizures, ataxia, dysarthria, or permanent memory } \\
\text { or mental changes. }\end{array}$ \\
3 & $\begin{array}{l}\text { Conscious, at least limited cognition, but dependent on others } \\
\text { for daily support. Ranges from ambulatory patients with severe } \\
\text { memory disturbances or dementia or paralysis. }\end{array}$ \\
4 & $\begin{array}{l}\text { Coma or vegetative state } \\
\text { Brain dead }\end{array}$ \\
\hline
\end{tabular}

Modified from Rittenberger et al. ${ }^{11}$

at time of hospital admission (Table 1). The neurologic/ cognitive status was quantified from admission and discharge notes according to the cerebral performance category (CPC) score using the same process described above for the GO-FAR score (Table 2). ${ }^{11}$ Good neurologic outcome was defined as $\mathrm{CPC}=1$ consistent with the two previous studies. Of note, HCMC has not contributed data to the Get With the Guidelines Registry, and thus, the data we report were not part of the database used to derive the GO-FAR score.

\section{Outcomes}

Pre-determined outcomes were survival to discharge, and survival to discharge with $\mathrm{CPC}=1$. Post hoc analyses include the association between these outcomes and timing of inhospital cardiac arrest (weekday vs weekend, before or after $48 \mathrm{~h}$ of hospitalization, and year of hospitalization).

\section{Statistical Analysis}

We used R (version 3.4.4) for receiving operator characteristic (ROC) analyses and Stata software (version 15.1; StataCorp) for all other analyses. Area under the curve calculations and comparisons were made using the pROC package, using the DeLong test (Xavier Robin, 2011). For comparisons of proportions, we used chi-squared test when expected frequencies were greater than 5 and Fisher's exact otherwise. We calculated confidence intervals using the Clopper-Pearson exact method. To reduce risk of type I error from multiple comparisons, our pre-determined alpha was set at 0.01 .

\section{RESULTS}

Demographic characteristics of our study population compared with those in the three previous studies using the GO-FAR score ${ }^{8-10}$ are shown in Table 3. Compared with these studies, our patients had a lower mean age (60.3 years, $95 \%$ CI 58.4 to 61.6$)$ and a slightly higher proportion were male $(65.5 \%, 95 \%$ CI 61 to $70 \%)$. In addition, our overall survival rate to discharge was significantly higher than the original US study ${ }^{8}$ (CID 14.5\%, 95\% CI 9.9 to $19.1 \%$ ) and the Skåne University study ${ }^{9}$ (CID $12.7 \%$, 95\% CI 6.2 to $19.3 \%$ ). Similar to the two previous external validation studies in Sweden, ${ }^{9,10}$ our proportion of patients with survival to discharge with good neurologic outcome (CID 7.0\%, 95\% CI 3.2 to $10.6 \%$ ) was significantly higher than reported in the original US study.

We compared our rates of survival with good neurologic outcome $(\mathrm{CPC}=1)$ grouped by GO-FAR score category with those in the Swedish and original derivation studies (Table 4). Survival to discharge with good neurologic outcome rates differed between GO-FAR survival groups (AUC 0.68, 95\% CI 0.62-0.73). Given the low number of patients to survive with $\mathrm{CPC}=1$ in our very low and low survival groups, we combined these two groups into a "below average" survival group as done in the previous validation study in Sweden. ${ }^{9}$ Of the 150 patients in this combined group, only $5.3 \%$ (95\% CI 2.3 to $10.2 \%$ ) survived to discharge with $\mathrm{CPC}=1$, significantly fewer than in the average $(22.5 \%, 95 \%$ CI 17.0 to $28.8 \%)$ or above average (34.1\%, 95\% CI 20.5 to $49.9 \%$ ) groups $(p<0.001)$. Similar to the external validation studies in Sweden, ${ }^{9,10}$ our survival rates were appreciably higher in the below average (CID 3.9\%, 95\% CI 0.3 to $7.5 \% ; p=0.002$ ) and average survival groups (CID 13.1\%, 95\% CI 7.4 to $18.8 \% ; p<0.001)$ compared with the original study.

Because survival following IHCA has been reported as worse when events occur on weekends, ${ }^{12}$ we evaluated whether we had disproportionately fewer IHCA occurring on weekends accounting for better outcomes than previously reported. Of 403 IHCA events, 293 (72.7\%, 95\% CI 68.4 to $77.1 \%)$ occurred on weekdays $(p=0.71)$. Of IHCA occurring on weekdays, survival to discharge with $\mathrm{CPC}=1$ was $18.7 \%$, compared with $13.6 \%$ of that on weekends (CID 5.1\%, 95\% $\mathrm{CI}-2.7$ to $13.0 \% ; p=0.24)$. The utility of the GO-FAR score calculated on admission was not significantly affected by weekday or weekend timing of IHCA (AUC 0.74 vs 0.65 ; $p=0.17$ ).

Because our study enrollment spanned 10 years, we also checked to determine whether a disproportionate number of IHCA occurred in the latter years of the study, given reported trends in increasing survival after IHCA over the past decade. ${ }^{1}$ Although more cases of IHCA occurred from 2014 to 2018 (232 compared with 171 from 2009 to 2013), overall discharge to survival was not statistically different (CID $1.5 \%, 95 \% \mathrm{CI}-10.7$ to $7.8 \% ; p=0.83$ ) nor was survival to discharge with $\mathrm{CPC}=1(\mathrm{CID} 3.3 \%, 95 \% \mathrm{CI}-4.2$ to $10.9 \%$; $p=0.42$ ) between the most recent 5 years and earlier years. Again, the GO-FAR score performed similarly in both groups (AUC 0.67 vs $0.68 ; p=0.87$ ).

Given a recent report that adults suffering IHCA within the first $48 \mathrm{~h}$ of hospitalization had better overall rates of survival to discharge and to discharge with good neurologic outcome ${ }^{13}$ we considered whether a disproportionate number of IHCA events occurred during the first $48 \mathrm{~h}$ of hospitalization. During our 10 -year study period, $44.6 \%$ of total IHCA events occurred within the first $48 \mathrm{~h}$ of hospitalization. Consistent with a recent report, ${ }^{13}$ a significantly higher proportion 
Table 3 Comparison of GO-FAR Study Populations' Characteristics

\begin{tabular}{lllll}
\hline \hline Characteristic & $\begin{array}{l}\text { Current study 2009- } \\
\mathbf{2 0 1 8}(\boldsymbol{n}=\mathbf{4 0 3})\end{array}$ & $\begin{array}{l}\text { Stockholm county }{ }^{\mathbf{1 0}} \mathbf{2 0 1 3}- \\
\mathbf{2 0 1 4}(\boldsymbol{n}=\mathbf{5 2 3})^{*}\end{array}$ & $\begin{array}{l}\text { Skåne University } \\
\mathbf{2 0 1 0}(\boldsymbol{n}=\mathbf{2 8 0 7})\end{array}$ & $\begin{array}{l}\text { Original US cohort }^{\mathbf{8}} \mathbf{2 0 0 7 -} \\
\mathbf{2 0 0 9}(\boldsymbol{n}=\mathbf{5 1 , 2 4 0})\end{array}$ \\
\hline Male gender, no. (\%) & $264(65.5)$ & $324(62)$ & $176(61.3)$ & $29,854(58)$ \\
Age, mean (SD), years & $60(16)$ & $71(14)$ & $70(\mathrm{NR})$ & $65(16)$ \\
$\begin{array}{l}\text { Survival to discharge, } \\
\text { no. } \%)\end{array}$ & $133(33)$ & $146(28) \dagger$ & $58(20)$ & $9479(18.5)$ \\
$\begin{array}{l}\text { Survival with CPC }=1, \\
\text { no. }(\%)\end{array}$ & $70(17)$ & $141(22)$ & $45(16)$ & $5329(10.4)$ \\
\hline
\end{tabular}

IHCA, in-hospital cardiac arrest; CPC, cerebral performance category

*Data for complete cases only

†30-day survival reported

of these patients survived to discharge $(39.4 \%, 95 \%$ CI 32.3 to $47.0 \%)$ than did those suffering IHCA after hospital day 2 (CID $11.6 \%, 95 \%$ CI 2.4 to $20.9 \% ; p=0.01$ ); and similarly, those with earlier IHCA had a higher incidence of discharge with good neurologic outcome $(23.3 \%, 95 \%$ CI 17.4 to $30.2 \%$ ) than did those suffering IHCA after hospital day 2 (CID $10.8 \%, 95 \%$ CI 3.2 to $18.3 \% ; p=0.005$ ). The GO-FAR score performed similarly regardless of timing of IHCA (AUC 0.68 vs $0.67 ; p=0.88)$.

\section{DISCUSSION}

This is the first confirmation of the potential value of the GOFAR score in a US hospital, which along with previous external validation studies in Sweden ${ }^{9,10}$ shows that the GO-FAR score is practicable for categorizing patients by probability of survival after IHCA with good neurological function (defined as CPC =1). Demonstration of the utility of the GO-FAR score at admission also indicates that this score, which can be easily calculated with an online tool (MDCalc) can be used at time of hospital admission when discussing the benefits and risks of CPR for IHCA. The GO-FAR score, in contrast to other prediction scores, uses variables that can be assessed prior to the IHCA event. Peri-arrest variables, such as the initial rhythm, duration of resuscitation, location, response time, and medications/interventions used, are highly predictive of outcomes, ${ }^{7}$ but are not knowable at the time when a clinician must discuss code status with a patient. The GO-FAR score was initially developed looking at some variables only in the immediate pre-arrest time period (Table 1). We deliberately determined whether these variables on admission were predictive of survival to discharge, given our goal of assessing prognosis at that time.

Both this study and the two external validation studies in Sweden ${ }^{9,10}$ showed significantly higher rates of survival with good neurologic function than reported in the original study (Table 4). We attempted to identify aspects of our study that might account for these differences. Although survival following IHCA has been reported as worse when events occur on weekends, ${ }^{12}$ we could not attribute our better survival to a disproportionate number of IHCA events on weekdays. Survival after IHCA has been improving over the past decade ${ }^{1}$ and our study evaluated IHCA occurring since 2009, whereas the GO-FAR score was developed from events from 2007 to 2009. However, despite increasing numbers of IHCA over the past 10 years, we found no significant difference in overall survival to discharge or survival to discharge with good neurologic outcome during the last 5 years compared to the previous 5 years.

Recently, Qazi et al. ${ }^{13}$ analyzed data in the Get With the Guidelines American Heart Association Resuscitation registry from 225,909 adults with IHCA between 2000 and 2014. In their study, $47.6 \%$ of IHCA occurred during the first $48 \mathrm{~h}$ of hospitalization, similar to $44.6 \%$ in our patients, and these patients had better overall rate of survival to discharge and to discharge with good neurologic outcome, as in our study. Of note, hospital day of IHCA was not reported in the three previous studies using the GO-FAR score. ${ }^{8-10}$ If a majority of events occurred during the first $48 \mathrm{~h}$ in the more recent studies, this might explain the increase observed survival for each GO-FAR group in those studies.

In both our study and the previous studies in Sweden,, 10 the percent of patients surviving to discharge with a good neurologic outcome was higher than in the original study, ${ }^{8}$

Table 4 Comparison of Rates of Survival to Discharge with CPC $=1$ by GO-FAR Score Group

\begin{tabular}{|c|c|c|c|c|c|}
\hline $\begin{array}{l}\text { GO-FAR } \\
\text { score } \\
\text { category }\end{array}$ & Survival group* & $\begin{array}{l}\text { Current study }(n= \\
403) \text {, survivors no. } \\
(\%)\end{array}$ & $\begin{array}{l}\text { Stockholm county, } \\
\text { Sweden }^{10}(n=532), \\
\text { survivors no. }(\%)\end{array}$ & $\begin{array}{l}\text { Skåne University, } \\
\text { Sweden }^{9}(n=287) \text {, } \\
\text { survivors no. }(\%)\end{array}$ & $\begin{array}{l}\text { Original cohort, } \mathrm{USA}^{8} \\
(n=51,240), \text { survivors } \\
\text { no. }(\%)\end{array}$ \\
\hline$>24$ & Very low & $3 / 60(5.0)$ & $1 / 38(2.6)$ & $1 / 26(3.8)$ & $14 / 1625(0.9)$ \\
\hline $\mathrm{T} 4-23$ & Low & $5 / 90(5.5)$ & $3 / 61(4.9)$ & $1 / 47(2.1)$ & $54 / 3247$ (1.7) \\
\hline$\geq 14$ & $\begin{array}{l}\text { Below average } \\
\text { (very low }+ \text { low) }\end{array}$ & $8 / 150(5.3)$ & $4 / 99(4.0)$ & $2 / 73(2.8)$ & $68 / 4872$ (1.4) \\
\hline-5 to 13 & Average & $47 / 209(22.5)$ & $40 / 258(15.5)$ & $12 / 147(8.2)$ & $869 / 9293(9.4)$ \\
\hline-15 to -6 & Above average & $15 / 44(34.1)$ & $97 / 166(58.4)$ & $31 / 67(46)$ & $839 / 3053(27.5)$ \\
\hline
\end{tabular}

*Likelihood of survival to discharge with $C P C=1$, labeled as "risk group" in previous studies 
especially for the patients of particular interest, those with a GO-FAR score of 14 or greater (the "very low" and "low" categories), which we and previous authors ${ }^{8,9}$ combined into a "below average" survival group. We found a clinically meaningful lower survival with good neurologic outcome in the below average group compared with the average (and above average) group. For providers who are looking to distinguish patients with a poor outcome after IHCA from other patients, our data combined with that from these previous studies ${ }^{8,9}$ would suggest that a GO-FAR score of 14 or greater corresponds to a likelihood of survival after IHCA with good neurologic outcome of approximately $5 \%$ or less.

\section{Study Limitations}

Our study had the inherent limitations of a single-site retrospective study. Our hospital has a unique patient population, availability of a full range of emergency and treatment services, and experienced physicians. Although we were able to collect data from 403 IHCA occurring over a 10-year interval, the number of observed events was small, especially in the below average survival likelihood group, similar to previous external validation studies. ${ }^{9,10}$

The GO-FAR score is highly dependent on assignment of CPC category. A CPC of 1 (neurologically intact or with minimal deficits) on admission will decrease the calculated GO-FAR score by 15 points, which corresponds to a 3-6-fold increase in predicted likelihood of survival with good outcome in all four studies. While widely used in research and quality assurance studies, most providers have little experience with and rarely use the $\mathrm{CPC}$ score in clinical practice. Consequently, all retrospective studies must determine the CPC score on admission and on discharge based upon the (often-limited) documentation provided in the medical record regarding cognitive and neurologic function. Not surprisingly, there is variable inter- and intra-reviewer agreement in extracting the CPC score retrospectively from medical records. ${ }^{14}$

The limitations of a single-site retrospective study with unique characteristics, improvements in management and outcomes after IHCA, and variability in assignment of CPC score may be responsible for varying discrimination of the GO-FAR score as measured by AUC. Our study had a notably weaker discrimination (AUC $0.68,95 \%$ CI 0.62-0.73) compared with the original derivation/validation study ${ }^{8}$ (AUC 0.78) and the Skåne University study ${ }^{9}$ (AUC $0.86,95 \%$ CI $0.82-0.9$ ). Future studies should prospectively evaluate the discrimination of the GO-FAR score in larger US hospital patient populations to validate this predictive tool further.

Finally, our study and the three previous GO-FAR studies had data only for patients who actually underwent CPR. Patients who already had a DNR order written would not be included in these studies, creating a systematic bias. For example, if patients with chronic incurable disease (renal disease, cirrhosis, metastatic cancer) and poor functional status more often had DNR orders, compared with those with these diseases but good functional status, the value of variables such as renal insufficiency, hepatic insufficiency, and metastatic cancer to predict survival with good neurologic outcome likely would be reduced. Further, incidences of DNR orders likely vary by hospital and patient population, ${ }^{15,16}$ and may be increasing over time. ${ }^{17-19}$

\section{CONCLUSIONS}

Most providers assume the responsibility for writing orders to initiate or withhold CPR when admitting a patient to the hospital without formal training in obtaining informed consent or informed refusal. ${ }^{3}$ In shared decision making, patients look to providers to supply information about prognosis and, after ascertaining a patient's values, to provide a recommendation about the proposed treatment. The code status discussion on admission to the hospital is typically suboptimal for multiple reasons, including that the patient is in crisis, does not know the provider, and has unrealistic expectations about outcomes after CPR, and the provider lacks information about survival and quality of life after CPR for IHCA. Typically, providers focus their conversation on the immediate risks of the procedure, such as breaking ribs, rather than survival, expected course of treatment, and quality of life after CPR.

Our study extends findings from three previous studies to show that the GO-FAR calculator (available online) can provide important information available at time of admission to the hospital to guide the informed consent discussion about CPR. Based upon these data, patients with a GO-FAR score of 14 or greater can be advised of very low likelihood (approximately 1 in 20) of surviving to hospital discharge being alert, able to work, and able to lead a normal life, and a 19 in 20 chance of either not surviving to discharge or surviving with some degree of cognitive disability. After eliciting the patient's values, providers can then give a recommendation for either a Full Code or a DNR status, emphasizing that the DNR status does not itself imply any limits on use of life-sustaining treatments while the patient has a pulse. Equally important, our results and those of a recent study ${ }^{13}$ suggest that providers should also readdress code status after $48 \mathrm{~h}$ of hospitalization, especially for patients who are Full Code and have a below average survival likelihood predicted by GOFAR score $>14$ upon admission, as likelihood for survival to discharge with good neurologic outcome is even worse if IHCA occurs on hospital day 3 or later.

Acknowledgments: The authors thank Dr. Drew Rosielle and Dr. Rebecca Sternschein for their thoughtful reviews of this manuscript.

Corresponding Author: Jeffrey B. Rubins, MD; Palliative Care Division, Hennepin Healthcare University of Minnesota, Minneapolis, MN, USA (e-mail: Jeffrey.Rubins@hcmed.org).

\section{Compliance with Ethical Standards:}

Conflict of Interest: The authors declare that they do not have a conflict of interest. 


\section{REFERENCES}

1. Benjamin EJ, Virani SS, Callaway CW, Chamberlain AM, Chang AR, Cheng S. Heart Disease and Stroke Statistics-2018 update: a report from the American Heart Association. Circulation. 2018; 137(5):e67-e492.

2. Silverman HJ, Tuma $\mathbf{P}$, Schaeffer $\mathbf{M H}$, Singh B. Implementation of the patient self-determination act in a hospital setting. An initial evaluation. Arch Intern Med. 1995; 155(5):502-10.

3. Binder AF, Huang GC, Buss MK. Uninformed consent: do medicine residents lack the proper framework for code status discussions? J Hosp Med. 2016;11(2):111-6.

4. Ebell MH, Bergus GR, Warbasse LA, Bloomer R. Failure of physicians to predict the outcome of in-hospital cardiopulmonary resuscitation. J Gen Intern Med. 1996;11(16):22.

5. Tomlinson $\mathbf{T}$, Brody $\mathbf{H}$, Tomlinson $\mathbf{T}$, Brody $\mathbf{H}$. Ethics and communication in do-not-resuscitate orders. N Engl J Med. 1988;318(1):43-6.

6. George AL, Folk BPI, Crecelius PL, Campbell WB. Pre-arrest morbidity and other correlates of survival after in-hospital cardiopulmonary arrest. Am J Med. 1989;87(1):28-34.

7. Ebell MH, Afonso AM. Pre-arrest predictors of failure to survive after inhospital cardiopulmonary resuscitation: a meta-analysis. Fam Pract. 2011;28(5):505-15

8. Ebell MH, Jang W, Shen Y, Geocadin RG. Development and validation of the Good Outcome Following Attempted Resuscitation (GO-FAR) score to predict neurologically intact survival after in-hospital cardiopulmonary resuscitation. JAMA Intern Med. 2013;173(20):1872-8.

9. Ohlsson MA, Kennedy LM, Ebell MH, Juhlin T, Melbye H. Validation of the good outcome following attempted resuscitation score on in-hospital cardiac arrest in southern Sweden. Int J Cardiol. 2016;221:294-7.

10. Piscator E, Göransson $\mathbf{K}$, Bruchfeld S, et al. Predicting neurologically intact survival after in-hospital cardiac arrest-external validation of the Good Outcome Following Attempted Resuscitation score. Resuscitation. 2018;128(July):63-9.
11. Rittenberger JC, Raina K, Holm MB, Kin YJ, Callaway CW. Association between cerebral performance category, modified Rankin scale, and discharge disposition after cardiac arrest. Resuscitation. 2011;82(8): 1036-40.

12. Robinson EJ, Smith GB, Power GS, et al. Risk-adjusted survival for adults following in-hospital cardiac arrest by day of week and time of day: observational cohort study. BMJ Qual Saf. 2016;25(11):832-41.

13. Gazi AH, Kennedy K, Bradley SM, Chan PS. Impact of timing of cardiac arrest during hospitalization on survival outcomes and subsequent length of stay. Resuscitation. 2017;121:117-22.

14. Ajam K, Gold LS, Beck SS, Damon S, Phelps R, Rea TD. Reliability of the cerebral performance category to classify neurological status among survivors of ventricular fibrillation arrest: a cohort study. Scand J Trauma Resusc Emerg Med. 2011;19:38.

15. Solveig A, Herlitz J, Bång A. Characteristics of patients who die in hospital with no attempt at resuscitation. Resuscitation. 2005;65(3):291-9.

16. Hospital variation in do-not-resuscitate orders and end-of-life healthcare use in the United States (letter). AnnalsATS. 2017;14(9):1485-9.

17. Phadke A, Heidenreich PA. Differences and trends in DNR among California inpatients with heart failure. J Card Fail. 2016;22(4):312-5.

18. Shih TC, Chang HT, Lin MH, Chen CK, Chen TJ, Hwang SJ. Trends of do-not-resuscitate orders, hospice care utilization, and late referral to hospice care among cancer decedents in a tertiary hospital in Taiwan between 2008 and 2014: a hospital-based observational study. J Palliat Med. 2017;20(8):838-44.

19. Richardson DK, Zive DM, Newgard CD. End-of-life decision-making for patients admitted through the emergency department: hospital variability, patient demographics, and changes over time. Acad Emerg Med. 2013;20(4):381-7.

Publisher's Note Springer Nature remains neutral with regard to jurisdictional claims in published maps and institutional affiliations. 